MODELING, IDENTIFICATION AND CONTROL, 1995, VOL. 16, NO. 2, 95-106

doi:10.4173/mic.1995.22

\title{
Input saturation in nonlinear multivariable processes resolved by nonlinear decoupling
}

\author{
JENS G. BALCHEN $\dagger$ and BJARNE SANDRIB $†$
}

Keywords: Multivariable control; saturation, nonlinear decoupling

\begin{abstract}
A new method is presented for the resolution of the problem of input saturation in nonlinear multivariable process control by means of elementary nonlinear decoupling (END). Input saturation can have serious consequences particularly in multivariable control because it may lead to very undesirable system behaviour and quite often system instability. Many authors have searched for systematic techniques for designing multivariable control systems in which saturation may occur in any of the control variables (inputs, manipulated variables). No generally accepted method seems to have been presented so far which gives a solution in closed form. The method of elementary nonlinear decoupling (END) can be applied directly to the case of saturation control variables by deriving as many control strategies as there are combinations of saturating control variables. The method is demonstrated by the multivariable control of a simulated Fluidized Catalytic Cracker (FCC) with very convincing results.
\end{abstract}

\section{Introduction}

The development of control strategies for multivariable nonlinear processes has high priority. In order that such control strategies shall be realistic, they must be able to cope with the cases when control variables (manipulated variables) reach saturation levels. No control strategy published so far has a closed form solution to this problem. Saturation in control variables may or may not be a serious problem. As an example, take a SISO-process which is unstable before it is stabilized through control by strong negative feedback via its control variable. If this control variable saturates the process will again become unstable. In multivariable processes the same situation may occur, but if the process is state controllable through more than one control variable, a systematic method for handling saturation may be developed. In control strategies based upon optimization and control vector parametrization (CVP) saturating control variables are easily handled (Biegler 1984, Balchen, Ljungquist and Strand 1992) but these solutions are not in closed form. Other attempts to resolve the problem are given and referenced in Singstad (1992) and Kothare, Campo, Morari and Nett (1993). The strategy to be developed below is based on the principle of elementary nonlinear decoupling (END) (Balchen 1993, Balchen and Sandrib 1994) which is a variation of the exact linearization technique (Isidori 1989). In END a property variable is introduced which becomes the object of control and which has as many degrees of freedom as the number of independent control variables. In the case of saturation in

Received 30 August 1994.

† Department of Engineering Cybernetics, The Norwegian Institute of Technology, N-7034 Trondheim, Norway.

(C) IEEE. This paper was presented at the 3rd IEEE Conference on Control Applications. 24-26 August 1994, Glasgow. Reprinted with permission. 
control variables the property variables are designed according to which control variables are saturated.

\section{The problem of saturating control variables}

The process to be controlled is in general described by a nonlinear model where

$x$ : state vector $(\operatorname{dim} x=n)$

$$
\dot{x}=f(x, u, v)
$$

$\boldsymbol{u}$ : control vector $(\operatorname{dim} u=r)$

$v$ : disturbance vector $(\operatorname{dim} v=p)$

$f(\cdot)$ : vector of nonlinear functions $(\operatorname{dim} f=n)$

Furthermore it is assumed that measurements from the processes are available through the relationship

$$
\boldsymbol{y}=h(\boldsymbol{x})+\boldsymbol{w}
$$

where

$\boldsymbol{y}$ : measurement vector $(\operatorname{dim} \boldsymbol{y}=m)$

$w$ : measurement noise vector $(\operatorname{dim} w=m)$

In the following it is assumed that a state estimation scheme in the form of an Extended (Augmented) Kalman Filter or something equivalent is implemented so that the whole state vector $(x)$ is available in the development of the control system.

A linearized version of (1) and (2) will be

$$
\begin{aligned}
& \delta \dot{x}=A \delta x+B \delta u+C \delta v \\
& \delta y=H \delta x+w
\end{aligned}
$$

where the matrices are appropriate Jacobian matrices of the nonlinear functions in (1) and (2).

The problem to be encountered is the situation which occurs when one or more of the control variables constituting $\boldsymbol{u}$ reach a saturation level. Such a situation is very realistic because physical variables definitely are limited in magnitude either caused by equipment constraints or safety considerations and these limits may be reached under normal disturbance conditions $(v)$ which are not necessarily severe.

When a control variable reaches a saturation value, either positive or negative, that particular variable seizes to influence the process behaviour by more than a constant value. The consequence is that the process has lost a degree of freedom in control. This may or may not have serious consequences depending on the structure of the function $f(\cdot)$ of $(1)$ or the matrix $B$ of (3).

The matrix $\mathbf{B}$ can be written in terms of parameter vectors $\boldsymbol{b}_{i}$ each representing the influence of the individual control variables $\left(u_{i}\right)$ upon the state equation of (30).

$$
\frac{\partial f(\cdot)}{\partial u}=B=\left[b_{1}, b_{2} \ldots b_{i} \ldots b_{r}\right]
$$

Therefore (3) can be written

$$
\delta \dot{\boldsymbol{x}}=A \delta \boldsymbol{x}+B^{i j \cdots} \delta u^{i j \ldots}+\boldsymbol{b}_{i} \delta u_{i s a t}+\boldsymbol{b}_{j} \delta u_{j s a t} \ldots+C \delta \boldsymbol{v}
$$

where $B^{i j \ldots}$ has been introduced for the matrix $\mathbf{B}$ where the columns $i, j \ldots$ are either removed or replaced by small numbers, $\delta u^{i j \ldots}$ is the control vector with elements $i, j \ldots$ 
removed and $\delta u_{i s a t}$ and $\delta u_{j s a t}$ represent the saturating values of the control variables number $i$ and $j$ respectively.

Elementary conditions for linear state controllability express that the system with saturating control variables $u_{i}, u_{j \ldots}$ is controllable if

$$
\operatorname{rank}\left[B^{i j \ldots}\left|A B^{i j \ldots}\right| \ldots\left|A^{n-1} B^{i j \cdots}\right|\right]=n
$$

Equation (7) can be tested for all possible combinations $B^{i j \ldots}$. If a non-controllable combination is discovered, the resulting system can be further analysed to see if the noncontrollability is detrimental or not (unstable etc.). If the noncontrollable modes are nondetrimental, a feedback control system can be implemented for each $B^{i j \ldots}$ based upon the estimated states $(\hat{\boldsymbol{x}})$ and the system will eventually return to normal nonsaturating conditions. If the noncontrollable modes are detrimental, there is usually no other way to solve the problem than by redesigning the process, for instance by adding more control variables.

\section{Elementary nonlinear decoupling (END)}

END is a version of nonlinear decoupling algorithms that solves the invertibility problem by designing the property space which is the object of linearization and decoupling. It has some similarity with the input-output linearization (Isidori 1989) in which the invertibility problem is solved by differentiating the output variables a number of times. In Balchen (1993) it is claimed that END is the most realistic solution in practical systems.

The objective of END is to derive the control vector $(\boldsymbol{u})$ for a nonlinear dynamic process described by (1) such that a property vector defined by the property transformation

$$
z=d(x)
$$

follows a certain desired trajectory defined by $\dot{z}=\dot{z}_{d \cdot}$. In most cases the property transformation of $(8)$ is replaced by a linear transformation

$$
z=D x
$$

where $D$ is a constant matrix.

One solution to the above problem is an iterative equation solver given by

$$
\dot{u}=K_{u}(\cdot)\left[\dot{z}_{d}-D f(x, u, v)\right]
$$

where the matrix $K_{u}(\cdot)$ secures convergence of (10). One way of securing the convergence of (10) is to require that the linearized loop described by (10) has prescribed and constant eigenvalues $(\Lambda)$ such that

$$
K_{u}(\cdot)=-\Lambda\left(D \frac{\partial f(\cdot)}{\partial u}\right)^{-1}
$$

A block diagram illustrating the END algorithm is shown in Fig. 1. It includes the nonlinear estimator which has an updating matrix denoted $K_{x}(\cdot)$ of unspecified form since a number of algorithms may be used.

Assuming for the sake of simplicity that (1) is replaced by (3) and the eigenvalues $\Lambda$ of (11) are very large the resulting system after the application of END will be 


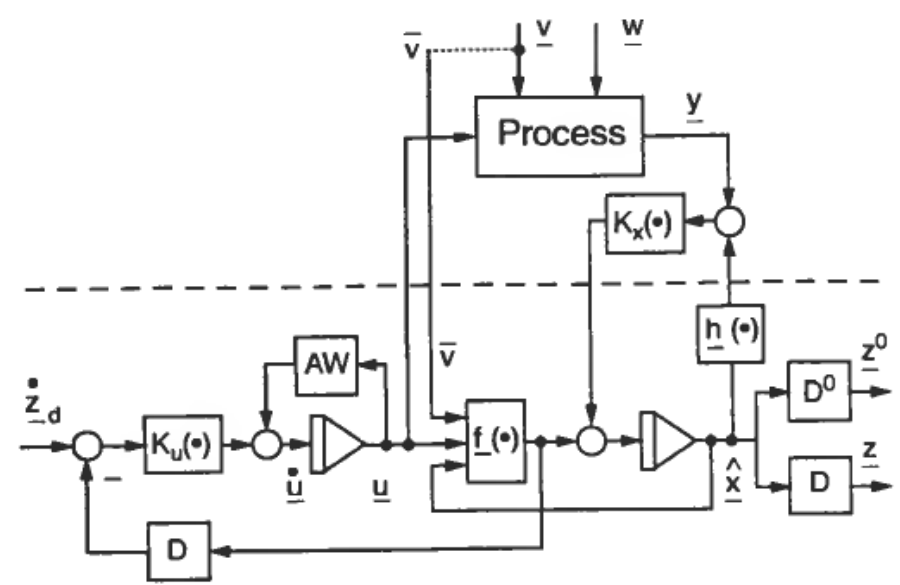

Figure 1. Block diagram of the END algorithm including nonlinear estimator.

described by

$$
\dot{\boldsymbol{x}}=\left(I-B(D B)^{-1} D\right)(A x+C v)+B(D B)^{-1} \dot{z}_{d}
$$

Multiplying (12) by $D$ yields

$$
\dot{z}=D \dot{\boldsymbol{x}}=\dot{\boldsymbol{z}}_{d}
$$

It is obvious from (11) that in order for a solution to exist the matrix (DB) must be non-singular. The least requirement for this to be true is that $\operatorname{dim} z=\operatorname{dim} u$.

Furthermore we must require that the dynamic system described by the state equation of (12) must have acceptable behaviour, i.e. eigenvalues properly located in the left half of the complex plane.

The dynamics of the system is determined by the differential equation of (12) with the eigenvalues of the matrix

$$
\left(I-B(D B)^{-1} D\right) A
$$

characterizing the system stability.

From (12) the main system transfer matrix is given by

$$
\boldsymbol{x}(s)=H(s) \dot{z}_{d}(s)
$$

where

$$
H(s)=\left(s l-\left(I-B(D B)^{-1} D\right) A\right)^{-1} B(D B)^{-1}
$$

This transfer matrix converted into the frequency response matrix $H(j \omega)$ can be characterized by the eigenvalues of (14) or by means of the maximal and minimal singular values $\bar{\sigma}_{H}(j \omega)$ and $\sigma_{H}(j \omega)$. This gives a method for designing dynamic behaviour by choosing different $D$ matrices so that the system becomes invertible with acceptable dynamic properties.

One could think of another transformation $z^{0}=D^{0} x$ which expresses the desirable but nonrealizable property vector. The reason for the nonrealizability is that the matrix $D^{0} B$ most often will become singular.

In the following design procedure $D$ is chosen in an unformal manner as a matrix which has only few elements different from those of $D^{0}$ such that the dynamic behaviour of the system characterized by the eigenvalues of (14) is acceptable. 


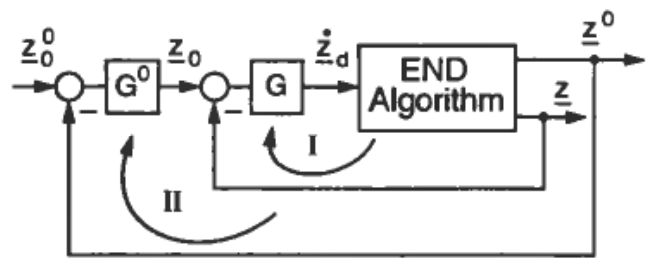

Figure 2. The control loops around the decoupled and linearized process.

In Fig. 2 is shown how feedback control is applied around the system which has been decoupled and linearized by means of the END algorithm. There is an inner loop (I) containing a control matrix $\mathbf{G}$ and with a reference vector $z_{0}$ and output $z$. When there is no saturation in any of the control variables, this loop will have nearly perfect response with very high bandwidth. Next there is an outer feedback loop (II) containing a control matrix $G^{0}$, a reference vector $z_{0}^{0}$ and an output $z^{0}$. This outer loop will be slightly degraded relative to loop $(I)$. In some cases the inner loop $(I)$ can be omitted yielding a high bandwidth performance.

\section{The end algorithm with saturating control variables}

When one of the control variables $\left(u_{i}\right)$ reaches saturation, the system looses one degree of freedom. This means that the number of property variables $(z)$ which can be controlled by the END algorithm must be reduced by one. In order to avoid introducing discontinuous jumps in the state variables of the process all but one of the property variables (rows of $D$ ) should be kept unchanged when a new saturation occurs. That is, one of the property variables must be sacrificed. The obvious choice in most cases is to sacrifice that property variable which has least significance in terms of 'survival' of the process rather than 'economy' of performance. This is so because saturation in one or more of the control variables is a transient phenomenon which will disappear after short time. If the saturation phenomenon does not disappear, there is something wrong in the design of the process which calls for major modifications.

In general the property vector associated with a certain set of saturating control variables symbolized by the associated control matrix $B^{i j \ldots}$ will be given by an associated property transformation matrix $D^{i j \ldots}$. The resulting system will have the same block diagram as that of Fig. 1 and the convergence matrix $K_{u}^{i j \ldots}(\cdot)$ will still be determined by the expression of (11) only with the new matrices applied.

In Fig. 1 the $r$ integrators determining the control vector $(u)$ must all be equipped with an 'anti wind-up'-facility securing that the integrators do not 'overcharge' when saturation occurs in either direction.

The procedure of the 'Saturating Elementary Nonlinear Decoupling' algorithm (SEND) will thus be as follows:

(1) A property transformation matrix $D^{i j \ldots}$ is chosen for each possible combination of saturating control variables. These matrices are stored in a data base.

(2) The corresponding matrices $K_{u}^{i j \ldots}(\cdot)$ algorithms are stored.

(3) Each control variable $\left(u_{i}\right)$ is tested to determine whether saturation has occurred, and the appropriate $D^{i j \ldots}$ and $K_{u}^{i j . \cdots}(\cdot)$ are chosen and implemented.

(4) Controllability analysis according to (7) should be performed for all possible combinations of saturating control variables to determine whether detrimental noncontrollability may occur. 


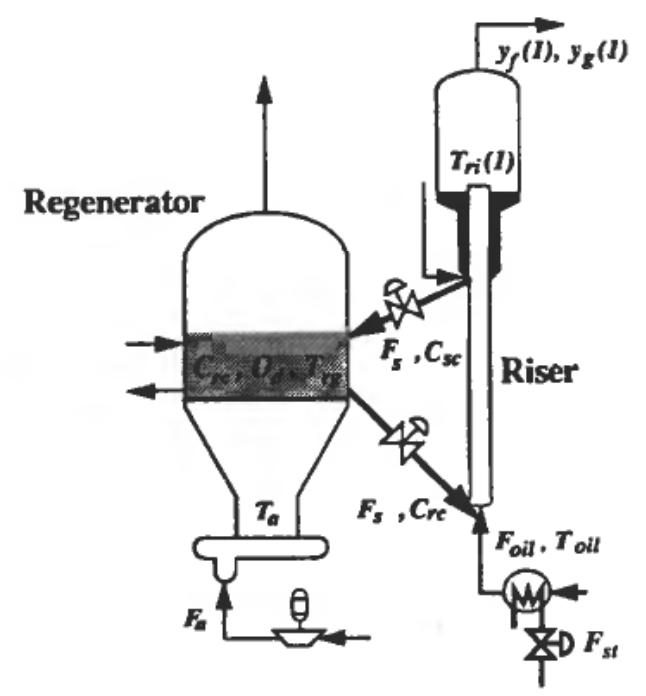

Figure 3. The simulated cracking plant.

Further discussion of the SEND algorithm will be given below in relation to the control of an FCC-process.

\section{The fluidized catalytic cracking process (FCC)}

A simplified diagram explaining the FCC process is shown in Fig. 3. It consists of a reactor part in the form of a riser in which the actual cracking process occurs and a regenerator. In the riser preheated oil consisting mainly of heavy hydrocarbons meets a flow of very hot catalyst material coming from the regenerator. The regenerator in turn receives the spent catalyst from cyclone separators on the top of the riser. In the regenerator (which may have one or two stages, in this case one) the carbon which has been deposited on the catalyst particles is burnt off by the supply of oxygen (air).

The FCC process is multivariable with a high degree of coupling between different state variables. A simplified, but quite accurate model of this process has been developed in Ljungquist (1990) and is reviewed in Appendix A. Five state variables are considered, namely

$x_{1}=C_{r c}$ : Coke on regenerated catalyst

$x_{2}=O_{d}:$ Oxygen in regenerator dense bed

$x_{3}=T_{r g}$ : Temperature in regenerator

$x_{4}=y_{8}(1)$ : Weight fraction of gasoline in product

$x_{5}=T_{\text {oil }}$ : Temperature in oil from preheater

Three control variables are assumed to be active, namely

$u_{1}=F_{s i}$ : Energy flow in steam to preheater

$u_{2}=F_{a}:$ Mass flow of air to regenerator

$u_{3}=F_{s}$ : Mass flow of regenerated catalyst

The main disturbances to be considered are

$v_{1}=k_{c}^{1}$ : Rate constant for catalytic coke formation

$v_{2}=F_{\text {oil }}$ : Mass flow of oil feed. 


\section{Simulation of SEND-algorithm applied to saturating $F C C$}

A large number of test runs have been made to evaluate the properties of the SEND-algorithm. Seven possible combinations of saturating controls exist:

$$
B, B^{1}, B^{2}, B^{3}, B^{12}, B^{13}, B^{23}, B^{123}
$$

where $B$ represents the unsaturated and $B^{123}$ the fully saturated case.

The following pairs of $D$ and $B$ matrices are tested

$$
\begin{gathered}
B \rightarrow D=\left[\begin{array}{l}
d_{1}^{\mathrm{T}} \\
d_{2}^{\mathrm{T}} \\
d_{3}^{\mathrm{T}}
\end{array}\right]=\left[\begin{array}{ccccc}
1 & 0 & 0 & 0 & 0 \cdot 01 \\
0 & 0 \cdot 1 & 1 & 0 & 0 \cdot 001 \\
0 \cdot 1 & 0 & 0 & 1 & -0 \cdot 015
\end{array}\right] \\
B^{1} \rightarrow D^{1}=\left[\begin{array}{l}
d_{1}^{\mathrm{T}} \\
d_{2}^{\mathrm{T}}
\end{array}\right], B^{2} \rightarrow D^{2}=D^{1}, B^{3} \rightarrow D^{3}=D^{1} \\
B^{12} \rightarrow D^{12}=d_{2}^{\mathrm{T}}, B^{13} \rightarrow D^{13}=D^{12}, B^{23} \rightarrow D^{23}=D^{12}
\end{gathered}
$$

As can be seen the $D^{i j \ldots}$ are all submatrices of the larger matrices. This is not a necessity but has the desirable feature that the state variables $(x)$ will not exhibit discontinuous jumps when switching the $D^{i j \ldots}$ matrices.

While the system is running (according to Fig. 1) it is continuously being tested to determine what combination of saturating control variables (according to (17)) that exists.

Since a mechanism is needed to bring the control variables out of saturation if possible, a search procedure is undertaken illustrated by the search tree

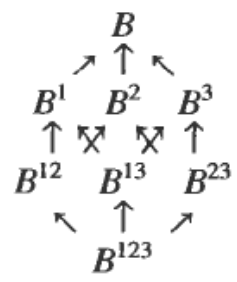

As an example:

If the system at present has $u_{i}$ and $u_{j}$ saturated (i.e. $B^{i j}$ ) a test is performed one level up in the search tree. The test checks the system at the boundary of the control variable range, i.e. first $B^{i}$ with $u_{j}=u_{j \max }$ and then $B^{\mathrm{j}}$ with $u_{i}=u_{i \max }$ to examine if these are 'possible' saturation states. A 'possible' saturation state $\left(B_{i}\right)$ is indicated when $u_{j}$ is leaving saturation when $B^{i}$ is tested and vice versa.

If the test finds that $B^{i}$ and $B^{j}$ are 'possible', the algorithm changes the current saturation search state from $B^{i j}$ to $B^{i}$ or $B^{j}$. If both $B^{i}$ and $B^{j}$ are 'possible' states a priority strategy must decide the choice. From $B^{i}$ (or $B^{j}$ ) a similar procedure finally brings the system to the top of the search tree $(B)$ which means no saturation in any control variable.

The saturation levels assumed for control variables can be read from the corresponding graphs. Two disturbance cases are studied:

- Soft step up and down of $k_{c}^{1}$ : Rate constant for catalytic coke formation

- Soft step up and down of $F_{\text {oil }}$ : Mass flow of oil feed 

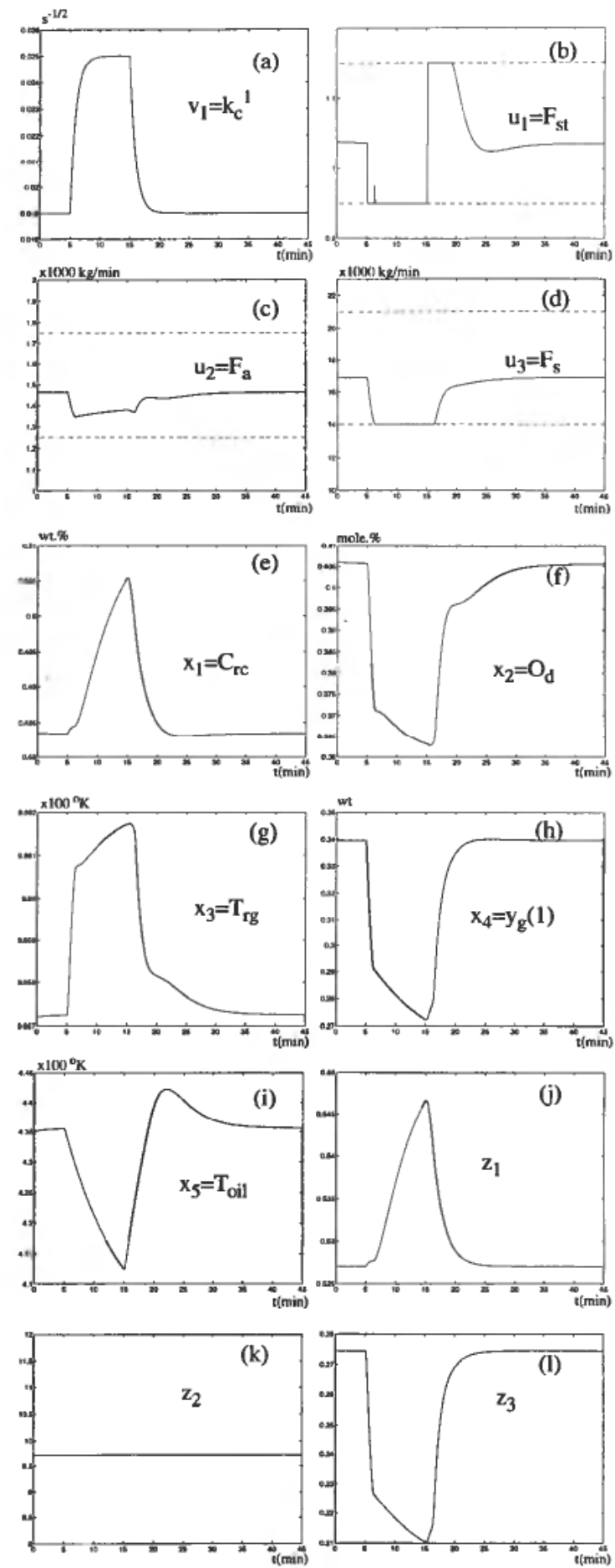

Figure 4. Responses of the FCC to changes in $k_{c}^{1}$ with saturation. 
The property transformation $D^{1}$ given above leads to the control of

$$
z_{1}=x_{1}+0.01 x_{5}=C_{r c}+0.01 T_{\text {oil }}
$$

and

$$
z_{2}=0 \cdot 1 x_{2}+x_{3}+0 \cdot 001 x_{5}=0 \cdot 1 O_{d}+T_{r g}+0 \cdot 001 T_{\text {oil }}
$$

whereas $D^{12}$ leads to control of only $z_{2}$ given in (22).

Figure 4 shows the system response to a 'soft' step in $k_{c}^{1}(t=5 \mathrm{~min}$ up, $t=15 \mathrm{~min}$ down). This change in $k_{c}^{1}$ leads to immediate saturation of $u_{1}$ at lower limit. Thus the system operates in state $B^{1} \rightarrow D^{1}$ with $z_{3}$ disregarded. At $t=7 \min u_{3}$ will also saturate at its lower limit, thus the system state changes from $B^{1} \rightarrow D^{1}$ into $B^{13} \rightarrow D^{13}$. At this state only $z_{2}$ is controlled via $u_{2}$.

At $t=15 \mathrm{~min} k_{c}^{1}$ is changed down again from 0.022 to 0.019 . The consequence of this is that $u_{1}$ changes from the lower to the upper saturation limit. The system is still in the state $B^{13} \rightarrow D^{13}$.

At $t \approx 16 \min u_{3}$ comes out of its saturation state and the system operates in state $B^{1} \rightarrow D^{1}$ with control of $z_{1}$ and $z_{2}$.

Finally at $t \approx 20 \min u_{1}$ also comes out of saturation and the system operates in state $B \rightarrow D$ with control of all the properties. The corresponding responses in the state variables and the property variables are shown in lower parts of Fig. 4. As can be seen the property $z_{2}$ which has been given priority does not change and the corresponding state $x_{3}=T_{r g}$ shows only a very small change $(0.04 \%)$. The uncontrolled properties show relatively small changes.

Similarly Fig. 5 shows the system response to a 'soft' step in $F_{o i l}(t=5 \mathrm{~min}$ up, $t=15$ min down). This change in $F_{\text {oil }}$ gives as result that $u_{1}$ goes into saturation at upper limit. Thus the system attains state $B^{1} \rightarrow D^{1}$ with $z_{3}$ out of control.

At $t \approx 8 \mathrm{~min} u_{2}$ nearly reaches saturation but since $u_{1}$ leaves saturation at about the same time the state changes to $B^{2} \rightarrow D^{2}$. This means that the system all the time operates with two control variables and therefore has the possibility of controlling two properties.

At $t=15 \mathrm{~min}$ when $F_{\text {oll }}$ changes in negative direction $u_{1}$ reaches its lower saturation limit whereas $u_{2}$ goes out of saturation. The consequence of this is that the state changes from $B^{2} \rightarrow D^{2}$ into $B^{1} \rightarrow D^{1}$ via $B^{12}$ but still controlling the properties $z_{1}$ and $z_{2}$.

At $t \approx 20 \mathrm{~min}, u_{1}$ will also come out of saturation and the system finally operates in state $B \rightarrow D$ with control of all the properties.

The responses in the states and properties are shown in the lower part of Fig. 5 again demonstrating that the control of the priority property $z_{2}$ and $x_{3}=T_{r g}$ is very good.

\section{Conclusions}

The principle of elementary nonlinear decoupling (END) has been shown to offer a direct approach to determining a control strategy when control variable saturation occurs in multivariable processes. One of the features of the method is that the designer must choose which properties to deemphasize through the choice of $D^{i j \ldots}$ when degrees of freedom in control are lost due to control saturation. 

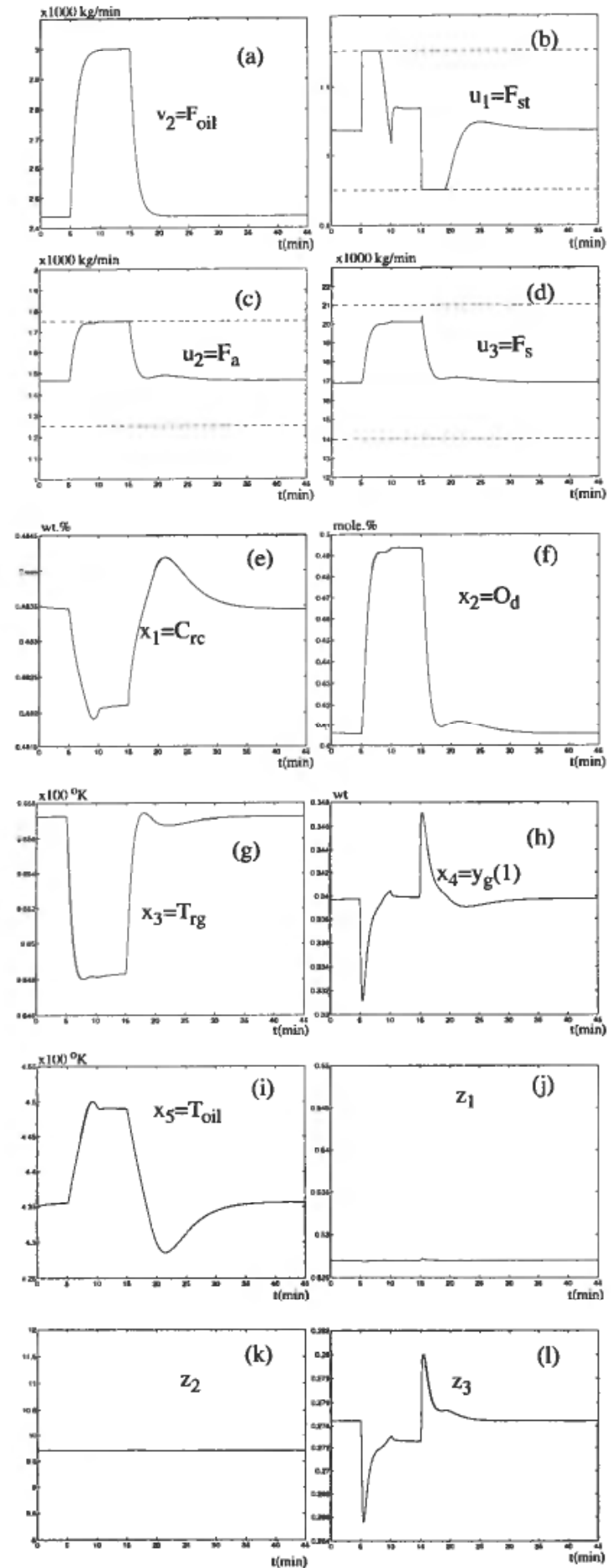

Figure 5. Responses of the FCC to changes in $F_{\text {oil }}$. with saturation. 


\section{ACKNOWLEDGMENT}

The research reported here has been partly supported (BS) by STATOIL and (JGB) by SINTEF.

\section{Appendix A}

The mathematical model of the FCC of Fig. 3 is taken from Ljungquist (1990) with some minor modifications. These modifications are:

(a) The riser dynamics are not neglected as in Ljungquist (1990) but replaced by a first order diff. equation (state $x_{4}=y_{8}(1)$ ).

(b) The dynamics of the oil preheater are not neglected as in Ljungquist (1990) but replaced by a first order diff. equation (state $x_{5}=T_{o i l}$ ).

The state space model becomes:

Regenerator:

$$
\begin{aligned}
W \frac{d C_{r c}}{d t} & =F_{s}\left(C_{s c}-C_{r c}\right)-k O_{d} C_{r c} W \\
W \frac{d O_{d}}{d t} & =R_{a}\left(O_{i n}-O_{d}\right)-\frac{n+2+(n+4) \sigma}{4 M_{c}(1+\sigma)} k O_{d} C_{r c} W \\
W c_{p s} \frac{d T_{r g}}{d t} & =T_{r i}(1) F_{s} c_{p s}+T_{a} F_{a} c_{p a}-T_{r g}\left(F_{s} c_{p s}+F_{a} c_{p a}\right)-\Delta H \frac{k O_{d} C_{r c}}{M_{c}} W \\
C_{s c} & =C_{r c}+C_{c a t} \\
k & =k_{c o m} \exp \left(\left(\frac{1}{960}-\frac{1}{T_{r g}}\right) \frac{E_{c b}}{R}\right) \\
\Delta H & =-h 1-h 2\left(T_{r g}-960\right)^{2} \\
\sigma & =1.1+\sigma_{2}\left(T_{r g}-873\right) \\
c_{p a} & =1.074
\end{aligned}
$$

Riser:

$$
\frac{d y_{g}(1)}{d t}=-10 y_{g}(1)+\frac{F_{g l}}{1-I_{g l}}\left(y_{f}(1)^{I_{g l}}-y_{f}(1)\right)
$$

where

$F_{g l}:$ gasoline yield factor of catalyst $\approx 1$

$I_{g l}$ : gasoline recracking intensity $\approx 0.9$

$y_{f}(1)$ : weight fraction of gasoil in product

and the equations for computing these variables are given in Ljungquist (1990).

Preheater:

$$
\frac{d T_{\text {oil }}}{d t}=-0 \cdot 1\left(T_{o i l}-T_{o i l, i n}\right)+42 F_{s t}
$$




\section{REFERENCES}

BALCHEN, J.G. (1993). Design of the property transformation in elementary nonlinear decoupling of multivariable processes, Modeling, Identification and Control, 14, 219-227.

BALCHEN, J. G. and SANDRIB, BJ. (1994). Elementary nonlinear decoupling control of composition in binary distillation columns, IFAC Symp. ADCHEM'94, Kyoto, Japan, May 1994.

BAlChen, J. G., LJungeuist, D. and STRAND, S. (1992) State-space predictive control, Chemical Engineering Science, 47, 787-807.

BIEGLER, L. T. (1984). Solution of dynamic optimization problems by successive quadratic programming and orthogonal collocation, Comp. Chem. Engn., 5, 243-248.

IsIDORI, A. (1989). Nonlinear Control Systems (2nd ed.) (Springer Verlag, Berlin).

Kothare, M. V., CAMPO, P. J., MoraRi, M. and NetT, C. N. (1993). A unified framework for the study of anti-windup designs, AIChE Annual Meeting, St. Louis, Paper no. 149d.

LJUNGQUIST, D. (1990). On-line estimation in nonlinear state-space models with application to catalytic cracking, Dr.ing Thesis, Department of Engineering Cybernetics, The Norwegian Institute of Technology, Trondheim, Report ITK-1990:89W.

SINGSTAD, P. (1992) Modelling and multivariable control of high pressure autoclave reactors for polymerization of ethene. Department of Engineering Cybernetics, The Norwegian Institute of Technology, Trondheim. Report ITK-1992:29-W. 